\title{
Article
}

\section{Exploratory evaluation of muscle strength and skin surface temperature responses to contemporary cryotherapy modalities in sport}

Alexander, Jill, Selfe, James, Greenhalgh, Olivia and Rhodes, David Available at http://clok.uclan.ac.uk/37644/

Alexander, Jill ORCID: 0000-0002-6492-1621, Selfe, James, Greenhalgh, Olivia and Rhodes, David ORCID: 0000-0002-4224-1959 (2021) Exploratory evaluation of muscle strength and skin surface temperature responses to contemporary cryotherapy modalities in sport. Isokinetics and Exercise Science, 29 (4). pp. 403-411. ISSN 0959-3020

It is advisable to refer to the publisher's version if you intend to cite from the work. http://dx.doi.org/10.3233/ies-200253

For more information about UCLan's research in this area go to http://www.uclan.ac.uk/researchgroups/ and search for <name of research Group>.

For information about Research generally at UCLan please go to http://www.uclan.ac.uk/research/

All outputs in CLoK are protected by Intellectual Property Rights law, including Copyright law. Copyright, IPR and Moral Rights for the works on this site are retained by the individual authors and/or other copyright owners. Terms and conditions for use of this material are defined in the policies page. 


\title{
Exploratory evaluation of muscle strength and skin surface temperature responses to contemporary cryotherapy modalities in sport
}

\author{
Jill Alexander ${ }^{\mathrm{a}, *}$, James Selfe ${ }^{\mathrm{b}}$, Olivia Greenhalgh ${ }^{\mathrm{a}, \mathrm{b}}$ and David Rhodes ${ }^{\mathrm{c}}$ \\ ${ }^{a}$ Sport, Nutrition and Clinical Sciences, School of Sport and Health Sciences, University of Central Lancashire, \\ Preston, UK \\ ${ }^{\mathrm{b}}$ Health, Psychology and Communities, Manchester Metropolitan University, Manchester, UK \\ ${ }^{C}$ Institute for Coaching and Performance (ICaP), School of Sport and Health Sciences, University of Central \\ Lancashire, Preston, UK
}

Received 20 November 2020

Accepted 25 March 2021

\section{Abstract.}

BACKGROUND: The effects of contemporary cryo-compression devices on function are limited compared to traditional applications of cooling. Development of cooling protocols are warranted.

OBJECTIVE: To investigate the effects of three different cryo-compressive modalities applied at the knee on the isokinetic strength of the quadriceps over a re-warming period.

METHODS: Eleven healthy male participants took part $(23 \pm 14$ years; $78.3 \pm 14.5 \mathrm{Kg} ; 180 \pm 9.5 \mathrm{~cm})$ randomly assigned to receive all modalities (Game Ready ${ }^{\circledR}$ (GR), Swellaway ${ }^{\circledR}$ (SA), Wetted Ice (WI)) applied for 15-min, separated by 1-week. Skin surface temperature $\left(\mathrm{T}_{s k}\right)$ via thermography and the concentric peak moment $(\mathrm{PM})$ of the quadriceps at 60 and $180^{\circ} / \mathrm{s}$ were collected pre-, immediately-post and at 20-min post-intervention.

RESULTS: Significant reductions occurred in $\mathrm{T}_{s k}$ across all timepoints for all modalities $(p=\leqslant 0.05)$. Significant reductions in PM for WI were noted across all timepoints and PM for GR and SA immediately-post $(p=\leqslant 0.05)$ only.

CONCLUSION: Precaution for immediately returning to sport following cryotherapy is required and influenced by type of cooling on muscle strength responses. Alternate targeted treatment modalities to minimise deferred deleterious effects on muscle strength may be considered. Research into length of application, periodisation and location is warranted for the development of such contemporary cryo-compressive modalities in applied practice.

Keywords: Cooling, knee, isokinetic dynamometry, performance, sport injury

\section{Introduction}

It is commonplace within sport to see ice being applied at varying times throughout competition to enable the continuation of competitive play. Aetiologi-

*Corresponding author: Jill Alexander, Sport, Nutrition and Clinical Sciences, School of Sport and Health Sciences, University of Central Lancashire, Preston, UK. Tel.: +44 1772 892781; E-mail: JAlexander3@uclan.ac.uk. cal risk factors strongly associated with non-contact musculoskeletal injury at the knee include reductions in strength [1-3]. Research has detailed the immediate detrimental effect icing protocols may have on muscle strength $[4,5]$ and knee joint kinematics [6,7]. These detrimental effects being shown to exist for $>20$-min post application $[5,6]$. Evidencing that during competition, such as half-time, consideration needs to be given to the potential injury risk exposure an athlete may be subjected to post ice application. This provides sports 
medicine practitioners with the dilemma of immediate therapeutic advantages, against potential increased or further injury risk. Contemporary pneumatic cryocompression devices aim to replicate similar reduced skin surface temperatures $\left(\mathrm{T}_{s k}\right)$ of between $10-15^{\circ} \mathrm{C}$, such as those induced by wetted ice (WI) [8], and although investigations into technological advances in cooling devices are acknowledged [9], the effects of such modalities on biomechanical function are limited within the available literature.

Historically, literature compares traditional cooling modalities such as gel packs, cold spray, crushed ice, frozen peas and WI [10-12]. Although, modern devices that combine cooling capabilities in conjunction with compression, such as the GameReady ${ }^{\circledR}$ (GR), Squid Go ${ }^{\circledR}$ and AIRCAST ${ }^{\circledR}$ Cryo/Cuff have gained prominence in the literature [8,13-15]. Evidence considers the addition of compression alongside cooling more effective at reducing $\mathrm{T}_{s k}[13,16]$, increasing oxygenation within tendons [17] and muscle [13], and the magnitude of cooling $[13,18]$, compared to the application of cooling alone. The comparison of contemporary cryo-compressive modalilties with intermittent vs consistent pressure demonstrated equivalent intramuscular temperatures decreases during applications [15], interestingly differences in $\mathrm{T}_{s k}$ were reported between modalities [15]. Similar findings are recently reported [13] for $\mathrm{T}_{s k}$ following varying levels of pressure and considered to be a key mechanism response for physiological processes to occur. Despite the known impact on physiological measures that additional compression influences, studies are still negligible that compare contemporary cooling devices with compression capabilities on strength parameters. This is most likely due to the lack of consensus individually for optimal cryotherapy or compression, including the length of application (minutes), which informs the rationale for the combined therapeutic use of cryo-compressive devices. Consequently, agreement as to what may be the 'optimum' application of such cryo-compressive devices are not yet determined. Contemporary developments in electronic pneumatic cryocompressive devices include Swellaway ${ }^{\circledR}$ (SA) (Swellaway.com), claimed to be a targeted local cooling device, and the GR (GameReady.co.uk), which provides intermittent compression with circumferential cooling.

Physiological measures of $\mathrm{T}_{s k}$ through infrared thermography (IRT) and functional strength quantifications of the lower limb musculature, via isokinetic dynamometry (IKD) support the understanding of potential effects local cryotherapy may have on objective mark- ers of function $[5,18,19]$. The relationship between reduction of $\mathrm{T}_{s k}$ and intramuscular temperature $\left(\mathrm{T}_{i m}\right)$ is previously reported [11], with a suggested quadratic relationship aiding to the understanding of the potential strength changes as reported in some studies following local cryotherapy applications $[5,10,19,20]$. Varying metrics have been utilised within literature to quantify muscle strength, including peak moment (PM) $[1,3,5]$. Utilisation of PM metrics provides practitioners with an overview of the athletes peak strength within one degree of movement. The effects of icing on biomechanical function are evident, yet not fully understood in relation to PM when comparing multiple cooling modalities. Methodologies differ however in the location of cooling application on mechanical properties of muscle and strength responses, with some studies applying only distal joint cooling [21,22], and others directly over soft tissue structures [23]. Recent literature reported that regardless of cooling location (quadriceps vs knee joint cooling) concentric muscle strength of the quadriceps decreased after exposure to WI application [5]. Further understanding however of contemporary cryo-compressive devices such as the SA and GR and their effects on biomechanical mechanisms, may support the progression of evidence necessary in helping to define optimum cryotherapy protocols for sports injury rehabilitation and recovery. Therefore, the aim of the present study is to compare the effect of cooling through the applications of WI, SA and GR on $\mathrm{T}_{s k}$ and the PM.

\section{Methods}

\subsection{Participants}

A priori power calculation was conducted using familiarisation trials and a minimum sample size of $\geqslant 10$ participants was required to evaluate the interactions associated with all independent variables (for statistical power $>0.8 ; P=<0.05$ ). The study was advertised through the University of Central Lancashire, and those volunteers that met the inclusion criteria of playing semi-professional rugby (classified as National 1 or 2 for the purpose of this study) with weekly training and competitive fixtures, alongside their university sports commitments were recruited. Consequently eleven healthy male participants met the criterion and volunteered to take part in the study (age: $23 \pm 14$ years; weight: $78.3 \pm 14.5 \mathrm{Kg}$; height: $180 \pm 9.5 \mathrm{~cm}$; body fat: $22.1 \pm 4.7 \%$ ). All experiments were undertaken with 
J. Alexander et al. / Exploratory evaluation of muscle strength and skin surface temperature responses

Table 1

Descriptive data for $\mathrm{T}_{s k}$ and $\mathrm{PM}$ for both speeds $\left(60^{\circ} . \mathrm{s}^{-1} ; 180^{\circ} . \mathrm{s}^{-1}\right)$ and across all timepoints

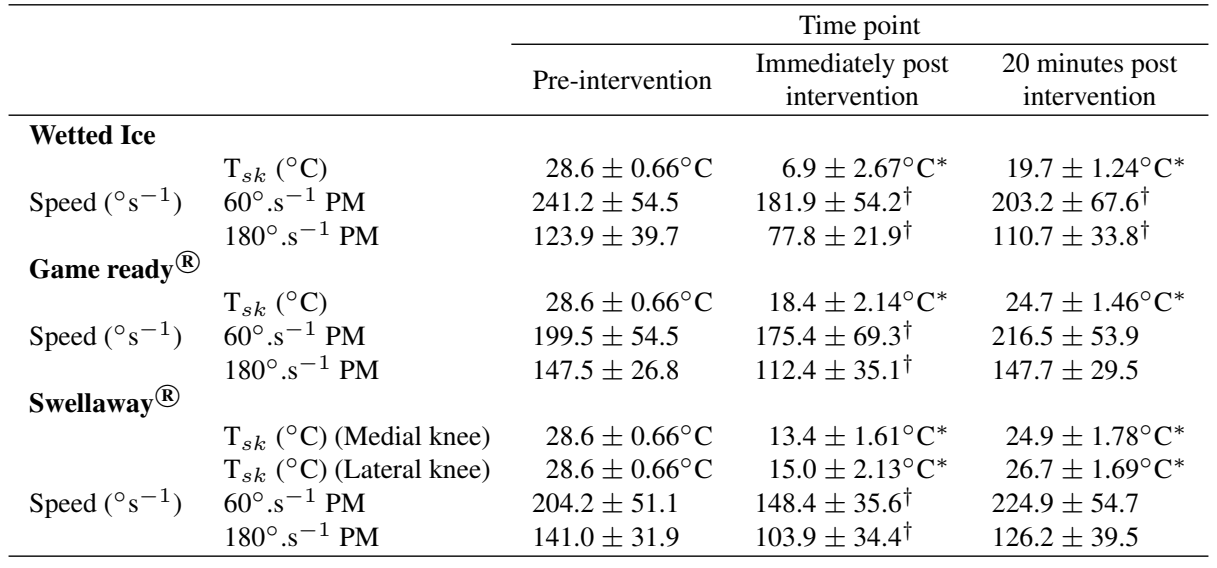

* Statistically significant reduction in $\mathrm{T}_{s k}$ post intervention. ${ }^{\dagger}$ Statistically significant reduction in concentric strength post intervention.

the understanding and written consent of each subject, and the study conformed with The Code of Ethics of the World Medical Association (Declaration of Helsinki, 2013), approved by the host university ethics committee (STEMH: approval date: September 2019). All male participation increased sample homogeneity due to gender differences found in response to local cooling [24]. Criteria for exclusion from this study included; females; any male under 18 years, history of musculoskeletal or neuromuscular lower limb injury in previous 6 months, any contraindications to cryotherapy [7,25]. All data was collected at pre-intervention, immediately postintervention and at 20-min post-intervention.

Participants were randomly assigned (randomization.com) to a sequence of cryotherapy modalities, separated by 1 week, which included the GR (GameReady ${ }^{\circledR}$, Global, UK), which provides cyclical pneumatic cooling through water and ice with simultaneous compression through manual protocol combinations; SA (Swellaway Ltd, UK) a pneumatic portable device providing simulataneous targeted cooling through Peltier cell technology and compression through pre-set protocol combinations; and WI made up from comprised of $800 \mathrm{~g}$ of crushed ice mixed with $800 \mathrm{ml}$ of water. Each modality followed a typical clinical application times of 15-min dosage [26], standardising common practices of cryotherapy applications in sport. Anthropometric data, including skin fold measurements (Harpenden Skinfold Callipers, HSB-BI; Baty International, Burgess Hill, West Sussex, UK), to estimate the percentage body fat based on the sum of skin fold thickness for adipose tissue measurements and were taken from the following sites: abdomen, me- dial calf, thigh, biceps, triceps, iliac crest, supraspinatus and subscapularis [27]. Records of dominant limb was quantified following previous published methods to determine favoured kicking foot [28] and ambient room temperature established during a 15-min acclimatisation period ensured continuity between baseline data protocols and participants prior to cooling intervention following previous methods [29]. Room temperature measured every 30-min throughout testing noted any fluctuations in environmental temperature.

\subsection{Testing protocol}

Skin surface temperature via a non-invasive thermal imaging camera (FLIR C3, FLIR ${ }^{\circledR}$ Systems, Inc.) and concentric isokinetic quadriceps strength (Cybex, Division of Lumex Inc., Ronkonkoma, NY, USA) established relevant objective biomechanical and physiological markers for the study. An accurate and reliable measure of $\mathrm{T}_{s k}$ can be quantified through thermal imaging [20,29], and in the current study this provided the opportunity to determine whether differences in $\mathrm{T}_{s k}$ exist between the three modalities when applying the same length of exposure (15-min) (Table 1). A ThermoVision A40M Thermal Imaging Camera (Flir systems, Danderyd, Sweden) with emissivity set at 0.97-0.98 was used according to standard medical protocols for the use of Thermographic Imaging in Sport and Exercise Medicine (TISEM) [29]. Mounted perpendicular to the defined region of interest (ROI) over the anterior, medial and lateral aspects of the knee, on a tripod at height $53 \mathrm{~cm}$; the TI camera connected to a laptop running Thermacam Researcher Pro 2.8 software (Flir 
systems, Danderyd, Sweden). To define the ROI over
the knee, thermally inert markers developed a suitable
framework for each modality application. Markers to
define a ROI for WI and GR applications were placed at
the tibial tuberosity, lateral and medial border of patella
at the same level with the tibiofemoral joint line, mid-
dle of the base of the patella [12]. Markers to define
a ROI for SA were placed at tibial tuberosity, lateral
and medial border of patella at the same level with the
tibiofemoral joint line, medial and lateral epicondyles
of the femur, and the medial and lateral condyles of
the tibia. Three images were taken at each timepoint
(Pre; Immediately post intervention; 20-min post in-
tervention) for each modality, with mean \pm SD T $\mathrm{T}_{s k}$
established during analysis.

The dynamometer was calibrated prior to each testing session. The data was collected at the same timepoints of baseline, immediately post intervention and 20-min post intervention and achieved by subjects performing five repetitions of knee extension at 60 and $180^{\circ} \mathrm{s}$ on the dominant leg [22]. For each testing speed the gravity-corrected moment angle curve was analysed. Analysis was restricted to the isokinetic phase with angular sectors of $100^{\circ}$ of knee flexion and $0^{\circ}$ to extension and for each phase the PM was identified. Highest PM values from repetitions for each timepoint were utilised for analysis. The limb was passively moved into flexion at $10 \%$ setween repetitions with 60 second rest between the two sets. Participants received verbal encouragement during the strength assessments. Participant position was supported at the distal thigh, distal tibia and across the chest when seated on the IKD follows standard protocol for this measurement [30]. A $90^{\circ}$ hip and knee flexion position prior to the commencement of movement permitted the input axis of the dynamometer to align with the lateral femoral condyle of the testing limb. Positional settings were recorded for each participant to ensure standardisation between each timepoint of data capture and between the different modality exposures. Throughout testing each repetition was observed by the same researcher to ensure smooth effort was exerted by each participant [22].

Interventions were allocated to participants in a random order of exposure comprised of either the GR; applied over the knee via the anatomical lower limb wrap, set at intermittent high compression $(5-75 \mathrm{~mm}$ $\mathrm{Hg}$ ) of 3-minute inflation compression cycles followed by 1 -minute recovery deflation, with a target temperature manually set to $10^{\circ} \mathrm{C}$ target temperature on the device (Fig. 1); WI comprised of $800 \mathrm{~g}$ of crushed ice mixed with $800 \mathrm{ml}$ of water in a sealed bag, held in

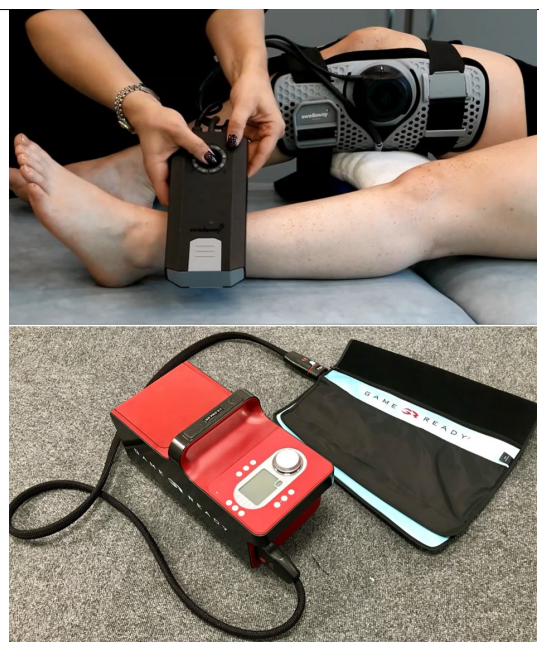

Fig. 1. Contemporary cryotherapy cooling modalities used in the study; the Game Ready ${ }^{\circledR}$ and the Swellaway ${ }^{\circledR}$ device.

place using standard cling wrap [10] over the anterior aspect of the knee; or the SA knee unit with $\times 2$ modular Peltier cells arranged medially and laterally over the knee joint line, held in place by a custom knee brace, with consistent pressure set at $35 \mathrm{~mm} \mathrm{Hg}$ and target temperature manually set to $10^{\circ} \mathrm{C}$ (Fig. 1). All cooling applications were applied with a standardised duration of 15-min [26]. Participants remained in a long sitting position on a medical plinth for the application of each cryotherapy modality. Following immediate removal of the intervention, thermographic images of the anterior knee and concentric quadriceps strength measures were taken following the same protocol as pre-intervention. The participant then returned to a long sitting position for a re-warming period of 20-min before further $\mathrm{T}_{s k}$ and the isokinetic moment measurements of the quadriceps were repeated. The choice to test at 0 and 20-min post-intervention was thought to replicate shorter time periods where athletes may return to functional competitive activity after exposure to cooling and timepoints representing previously published methodologies $[5,7]$. During the rewarming period, participants were encouraged to remain as still in the same position throughout this time. Participants returned at 1 and 2 weeks via randomisation for exposure to all modalities in the study.

\subsection{Analysis}

Statistical analysis was completed using PASW Statistics Editor 26.0 for Windows (SPSS Inc, Chicago, IL). A univariate, repeated-measures general linear 
model quantified the main effects for recovery duration following cooling interventions and for all isokinetic testing speeds. Significant main effects in recovery duration were explored using post-hoc pairwise comparisons with a Bonferroni correction applied. To ensure model adequacy the assumptions associated with the statistical model were assessed and met. Mauchly test of sphericity was also completed for all dependent variables, with a Greenhouse-Geisser correction applied if the test was signficant. To estimate effect sizes for all signficant main effects partial eta-squared $\left(\eta^{2} \mathrm{p}\right)$ values were calculated and classified as small (0.01-0.059), moderate $(0.06-0.137)$, and large $(>0.138)$ [31]. Interactions within the general linear model were identified within the analysis of data. Statistical significance was set at $P \leqslant 0.05$, and all data are presented as mean (SD).

\section{Results \\ 3.1. Skin surface temperature $\left(T_{s k}\right)$}

Skin surface temperature demonstrated statistically significant reductions immediately post cooling $(P<$ 0.05 ) and at 20-min post cooling for all three modalities $(P<0.05)$ compared to pre-application temperatures (Table 1). It did not return to baseline temperatures at 20-min post intervention for any of the three modalities (Table 1).

\subsection{Peak moment}

Table 1 summarises the effects of all cryotherapy modalities and the temporal pattern of recovery on PM. There was a significant main effect for speed postintervention $\left(P \leqslant 0.001, \eta^{2}=0.47\right)$ and time $(P \leqslant$ $\left.0.001, \eta^{2}=0.21\right)$, with no significant effect of modality found $\left(P>0.05, \eta^{2}=0.02\right)$. No significant 2-way or 3 -way interactions between the variables speed, time and modality were observed $(P>0.05)$ and therefore led to the examination of modalities separately for further exploration.

With the data set separated to consider effects of speed and time for each modality, significant main effects for time and speed for PM were identified for all three modalities (WI: time: $P=0.007, \eta^{2}=0.17$, speed: $P \leqslant 0.001, \eta^{2}=0.546$; GR: time: $P \leqslant 0.001$, $\eta^{2}=0.17$, speed: $P \leqslant 0.001, \eta^{2}=0.39$; SA: time: $P \leqslant 0.001, \eta^{2}=0.30$, speed: $P \leqslant 0.001, \eta^{2}=0.47$ ). No significant speed $\mathrm{x}$ time interaction were found
$(P>0.05)$ for PM. All modalities identified a significant reduction in strength immediately post intervention $(P<0.05)$, with only WI however demonstrating a significant reduction in strength 20-min post intervention $(P<0.05)$ for PM.

\subsection{Collapse of test speeds}

Analysis of the different speeds identified significant main effects for time $(P<0.001)$, but not for modality $(P>0.05)$ for $60^{\circ} \%$ and both time and modality for $180^{\circ} / \mathrm{s}(P<0.001)$ for PM. Observation of time points for PM for both speeds identified significant differences between time points pre and immediately post intervention $(P<0.001)$ and immediately post intervention and 20 -min post intervention $(P<0.001)$. Further analysis identified no significant differences in strength at $60^{\circ} \%$ between modalities for PM. Strength at $180^{\circ} / \mathrm{s}$ however identified significant differences between WI and GR $(P<0.001)$; WI and SA $(P<0.001)$; but not between GR and SA $(P>0.05)$.

\section{Discussion}

The purpose of the study was to investigate the effects of three different cryotherapy modalities on concentric knee extensor strength over a re-warming period. Findings report significant differences in the amount of PM output between GR, SA and WI modalities when applied to the knee for the same exposure period of 15 min. With WI having the greatest effect on strength reduction across multiple time points. Optimal cryotherapy protocols in sporting contexts are yet to be established. In consideration of the known immediate and latent effects on strength, a key aetiological risk factor associated with non-contact musculoskeletal injury [1-3], it is important to investigate and compare the biomechanical responses across different cryotherapy modalities to help best inform practical application in sport. This is the first study to the authors knowledge that compares contemporary cryo-compressive devices offering circumferentical or targeted applications to traditional WI methods. Results within the present study are largely consistent with previous work, displaying reductions within strength parameters immediately [5] and up to 20-min post cooling [22]. Yet, findings suggest the effects may be influenced through choice of cooling modality.

It was found that WI had the greatest effect on strength and for a longer period compared to the GR 
6 J. Alexander et al. / Exploratory evaluation of muscle strength and skin surface temperature responses

or SA devices, with significant reductions identified immediately and at 20-min post-intervention. Interestingly, when observing the two contemporary cryocompressive modalities utilised in this study (GR/SA) they were both associated with significant knee extension strength reductions immediately, but not at 20-min, post-intervention. It is evidenced that intramuscular temperatures continue to decrease after removal of cooling $[10,11]$. We might assume therefore that due to the efficacy of WI to conduct thermal energy this resulted in the significant reduction of strength at 20-min in the current study. A disruption to neuromuscular response through joint cooling previously suggests this may be a contributing factor to muscle strength loss in surrounding tissues such as the quadriceps [22]. Current data is representative of these presumptions and suggests that different neuromuscular responses occurred between modalities. Known physiological changes that ensue when therapeutic range is met, such as a reduction in nerve conduction velocity which influences joint receptor feedback [32] may not have been affected at 20-min post GR or SA applications. It is thought that this may be due to a) inadequate length of application exposure (time) to initiate response, and b) smaller, targeted region of joint cooling of the SA device compared to WI. The authors acknowledge literature that suggests exposure (time) should be ideally altered in consideration of adipose tissue levels [8] or target tissue [33] to induce physiological responses. In the current study however the same length of exposure (15-min) was applied across all particiants and between modalities to ensure standardisation of that particular variable, and was not the main focus of investigation in this study. Contrasting characteristics between modalities and the phasechange capability of material may advocate the results reported and contribute to the differences in strength output. Consequently this has implications on modality choice in terms of therapeutic treatment target.

Significant effects were observed in the current study for the speed of test, challenging findings in previous work questioning the use of a variety of speeds within research $[34,35]$. It is considered that higher velocity speeds more closely reflect the functional demands of multidirectional movements which require increased control at the knee to perform. Furthermore, injury risk is heighted through functional movements performed at higher velocity speeds. In the current study, at speeds of $180^{\circ}$ s, significant effects on PM for time and modality compared to only time for the lower speed of $60 \%$ s were reported. Reductions of strength noted at higher speeds compared to lower speed following the application of local knee joint cooling may suggest greater implications on functional control exist when observing PM parameters, and be influenced by modality choice. This requires further investigation, however practitioners might consider choice of cooling modality and length of rewarming periods to help mitigate the effects on functional control at the knee following local cooling applications.

Across all three modalities a typical re-warming curve in $\mathrm{T}_{s k}$ was noted, replicating earlier literature [25], with $\mathrm{T}_{s k}$ having not returned to baseline measures at 20-min post-intervention. When comparing devices on their ability to reduce $\mathrm{T}_{s k}$ differences were noted, and may therefore rationalise the differences in muscle strength changes observed in the current study. Notably $\mathrm{T}_{s k}$ reductions were significantly greater for WI compared to the GR or SA devices. In line with previous research, it is assumed from the reduction in strength at 20-min post removal of WI, intramuscular temperatures were more adversely affected compared to the GR or SA applications, of the same length of exposure (15-min). Current findings are representative of previous work that suggests that a quadratic relationship may exist between $\mathrm{T}_{s k}$ and $\mathrm{T}_{i m}$ post cooling [10] and that reductions in intra-articular temperatures may continue after the removal of cooling [36]. Although this is only an assumption as intra-articular temperature measures were not in the current study. Considering the continuing reductions possible in the intraarticular knee joint following removel of such cooling devices and applications; with therapeutic advantages of maintaining tissue temperature reductions, comes the increased potential for risk of further injury through reduced joint control, due to detrimental responses in surrounding muscle strength and meuromuscular responses to cooling [5,22]. WI cooled $\mathrm{T}_{s k}$ to a lower temperature, over a larger area compared to the SA device and as a result unsurprisingly negatively affected strength for a prolonged period post removal. The targeted cooling application of the SA device demonstrated the ability to reduce a local region of $\mathrm{T}_{s k}$ that was in direct contact with the Peltier cell, to within the therapeutic range $\left(10-15^{\circ} \mathrm{C}\right)[25]$ without diminishing PM 20-min post-application removal. It is important to note that a much smaller targeted area of cooling via the SA device may only be achieved without any indication of cooling spread in the adjacent skin away from the Peltier cell contact, in comparison to the other modalities, with cooling targeted over medial and lateral knee joint lines in the current study protocol. Although $\mathrm{T}_{s k}$ varied between medial and lateral locations (Table 1), 
$\mathrm{T}_{s k}$ reduced to within therapeutic range $\left(10-15^{\circ} \mathrm{C}\right)$ over both contact areas (medial and lateral knee joint lines) via Peltier cells, the cooling mechanism behind the SA device. This may be beneficial for targeted intervention applications, as no spread of cooling outside Peltier cell skin contact occurs, however as a result circumferential cooling cannot be achieved in comparison to the other modalities such as the GR or WI bags. Therefore the aim of treatment is important to identify prior to application of cooling modality choice. The rapid re-warming of $\mathrm{T}_{s k}$ observed by the SA modality may be advantageous in the development of identifying parameters of safer return to activity post cooling, as no detrimental muscle strength losses were reported at 20min post. It may be beneficial however to determine the effects of such device on joint kinematics, perception of pain and other physiological mechanisms to strengthen or dispute these findings further.

The 15-min exposure time application and target temperature setting of $10^{\circ} \mathrm{C}$ applied in the current study, reflecting pitch side or recovery applications of cooling appeared inadequate for the GR device to achieve a typical $\mathrm{T}_{s k}$ range of between $10-15^{\circ} \mathrm{C}$ immediately post removal (Table 1). Previous evidence demonstrates the ability of this device to cool to within therapeutic range [20] yet methodological differences in target temperature between studies as a rationale for the duifferences, appear to differentiate between agreement in results, which is unsurprising.

Despite similar presentations in strength responses for GR and SA devices at 20-min post it is assumed unlikely that detrimental changes in muscle strength would be reported for the GR. Previous literature applied an exposure time of 30-min for the GR device [14], but the authors felt this was not representative of shorter exposures of cryotherapy utilised in sport pitch-side, at half-time or intermittent stoppages in play, hoped to be investigated in the current study. The shorter application time for the GR device in the current study, reduced the capability of comparison to previous methodologies limiting evaluation of outcomes measured in the current study to others. Future investigations might observe longer periods of applications or cooler target temperatures for the GR $(\sim 30 \mathrm{~min})$ as represented in manufacturers' protocol options. This would aid as a dosage comparison and to ensure physiological changes, notably in analgesia are sufficiently induced. That said, the current findings highlight important differences in strength responses that therefore occur between cooling modalities of the same length of exposure (time). Current results suggest that both ben- eficial and disadvantageous differences arise between modalities, however as to which modality represents best practice is determined upon the aim of treatment and justification of use within the applied sport setting.

One application difference between the modalities in the current study was the adjunct of compression, that being manual-consistent pressure offered with WI (via plastic wrap), pneumatic-consistent pressure for the SA device, and pneumatic-intermittent pressure of the GR. Furthermore, the level of compression was not quantified and may be a limitation in the ability to justify the outcomes of the findings. It may be possible that the results of the GR were in fact due to the differences in compression adjunct (in term of intermittent vs consistent pressure) given that previous literature suggests it can produce similar levels of cooling compared to other cooling modalities [14,20]. Direct comparison to earlier studies is difficult due to differences in the modalities investigated. Further exploration of different methods of compression adjuncts offered by contemporary cooling modalities is warranted to understand the full extent of the impact it may have on physiological and biomechanical responses.

Choice of cryotherapy modality is an important part of clinical decision making. The phase-change capability of modalities is of note when comparing the efficacy of cryotherapeutic modalities, a panacea of variables influences this, including metabolic changes associated with injury, modality temperature and compressive adjunct, alongside length of exposure (time). Future research should consider the differences in material properties and length of exposure (time) of contemporary cooling applications on biomechanical functions. Subsequently the addition of eccentric muscle strength and joint position sense measures may be beneficial to develop a body of evidence around optimal protocols of contemporary cooling applications in sport. Sports medicine and performance practitioners should however continue to consider the differences and deleterious effects some applications of cooling may have on strength following lower limb cooling. A reduction in muscle strength and consequently performance suggests joint stability feedback mechanisms could also be compromised, as reported in previous literature [5,6]. Therefore, contemporary cooling devices that offer targeted local cooling, such as the SA device may be useful in comparison to circumferential joint cooling (GR) and vice versa, dependent on injury presentation, rehabilitation or recovery aim. The findings of the study are qualified by the homogeneous sample which limit the transferability of findings to female or injured populations. 
$\underline{8}$ J. Alexander et al. / Exploratory evaluation of muscle strength and skin surface temperature responses

Further research is required to address this issue and to provide sports medicine practitioners with evidenced based rationale for application of such modalities.

\subsection{Conclusion and practical implications}

Thermal imaging assessment for $\mathrm{T}_{s k}$ aided by isokinetic dynamometry continues to advance the development of applied guidelines for cryotherapy. Overall results are an important indication of the variation in effects on muscle strength response for several contemporary and traditional cryotherapeutic modalities. The results highlight the importance of acknowledging strength loss differences that occur between modalities following lower limb knee joint cooling. Precaution for athletes returning to functional activity following cryotherapy is required, and the extent of which is dependent on several biomechanical factors in need of further investigation simultaneously. Therapists may consider the following points when applying this modality:

- The effects of GameReady ${ }^{\circledR}$, Swellaway ${ }^{\circledR}$ and wetted ice on muscle strength measures and skin surface temperature observed over a rewarming period differ despite consistent length of exposure (time) applied (15-min).

- Contemporary cooling devices offering circumferential joint cooling (GameReady ${ }^{\circledR}$ ) may be useful in comparison to targeted local cooling (Swellaway ${ }^{\circledR}$ ) and vice versa, dependent on the aim of treatment.

- Targeted treatment using the Swellaway ${ }^{\circledR}$ device may minimise any deferred deleterious effects of cooling on specific muscle strength parameters.

- Further investigation is required to optimise application protocols using contemporary cooling devices compared to traditional methods for sport injury and recovery strategies.

\section{Acknowledgments}

The authors wish to thank the participants that volunteered to take part in the study.

\section{Author contributions}

CONCEPTION: Jill Alexander, James Selfe, Olivia Greenhalgh and David Rhodes PERFORMANCE OF WORK: Jill Alexander, Olivia Greenhalgh and David Rhodes
INTERPRETATION OR ANALYSIS OF DATA: Jill Alexander, James Selfe, Olivia Greenhalgh and David Rhodes PREPARATION OF THE MANUSCRIPT: Jill Alexander, James Selfe, Olivia Greenhalgh and David Rhodes REVISION FOR IMPORTANT INTELLECTUAL CONTENT: Jill Alexander, James Selfe, Olivia Greenhalgh and David Rhodes

SUPERVISION: James Selfe and David Rhodes

\section{Ethical considerations}

All experiments were undertaken with the understanding and written consent of each subject, and the study conformed with The Code of Ethics of the World Medical Association (Declaration of Helsinki, 2013), approved by the University of Central Lancashire ethics committee (STEMH: approval date: September 2019).

\section{Conflict of interest}

The authors have no conflict of interest to report.

\section{Funding}

The authors report no funding.

\section{References}

[1] Greig M. The influence of soccer-specific fatigue on peak isokinetic torque production of the knee flexors and extensors. Am J Sports Med 2008; 36: 1403-1409.

[2] Ekstrand J, Walden M, Hagglund M. Hamstring injuries have increased by $4 \%$ annually in mens professional football, since 2001: a 13-year longitudinal analysis of the UEFA elite club injury study. Br J Sports Med. 2016; 50: 731-737.

[3] Rhodes D, McNaughton L, Greig M. The temporal pattern of recovery in eccentric hamstring strength post-soccer specific fatigue. Res Sports Med. 2018; 273: 339-350.

[4] Hatzel B, Kaminski T. The effects of ice immersion on concentric and eccentric isokinetic muscle performance in the ankle Isokin Ex Sci. 2000; 8: 103-107.

[5] Alexander J, Rhodes D. Temporal patterns of knee extenso isokinetic torque strength in male and female athletes following comparison of anterior thigh and knee cooling, over a rewarming period. J Sport Rehab. 2019; 1-18.

[6] Alexander J, Richards J, Attah O, et al. Delayed effects of a 20-min crushed ice application on knee joint position sense assessed by a functional task during a re-warming period. Gai \& Posture. 2018; 62: 173-178. 
[7] Alexander J, Selfe J, Olivier B, et al. An exploratory study into the effects of a 20 minutes crushed ice application on knee joint position sense during a small knee bend. Phys Ther Sport. 2016; 18: 21-26.

[8] Alexander J, Rhodes D, Birdsall D, et al. Comparison of cryotherapy modality application over the anterior thigh across rugby union positions: a crossover randomised controlled trial. Int J Sports Phys Ther. 2020; 15: 210-220.

[9] Hawkins J, Schurtz J, Spears C. Traditional cryotherapy treatments are more effective than game ready ${ }^{\circledR}$ on medium setting at decreasing sinus tarsi tissue temperature in uninjured subjects. J Athl Enhance. 2012; 1: 1-5.

10] Dykstra JH, Hill, HM, Miller MG, et al. Comparisons of cubed ice, crushed ice and wetted ice on intramuscular and surface temperatures changes. J Athl Train. 2009; 44: 136-141.

11] Hardaker NJ, Moss AD, Richards $J$ et al. The relationship between skin surface temperature measured via non-contact thermal imaging and intra-muscular temperature of the rectus femoris muscle. Thermology Int. 2007; 17: 45-50.

12] Selfe J, Hardaker N, Whitaker J, et al. An investigation into the effect on skin surface temperature of three cryotherapy modalities. Thermol Int. 2009; 19: 121-126.

13] Alexander J, Greenhalgh O, Rhodes D. Physiological parameters in response to levels of pressure during contemporary cryotherapy applications: implications for protocol development. J Athl Enhanc. 2020; 9: 1-6.

14] Holwerda SA, Trowbridge CA, Womochel KS, et al. Effects of cold modality application with static and intermittent pneumatic compression on tissue temperature and systemic cardiovascular responses. J Athl Train. 2013; 5: 27-33.

15] Ostrowski J, Purchino A, Beck M, et al. Effectiveness of salted ice bag versus cryocompression on decreasing intramuscular and skin temperature. J Sport Rehab. 2019; 28: 120-125.

16] Merrick M, Knight K, Ingersoll CD, et al. The effects of ice and compression wraps on intramuscular temperature at various depths. J Athl Train. 1993; 28: 236-245.

[17] Knobloch K, Grasemann R, Spies M, et al. Midportion achilles tendon microcirculation after intermittent combined cryotherapy and compression compared with cryotherapy alone. A randomised trial. Am J Sports Med. 2008; 36: 2128-2138.

18] Tomchuk D, Rubley MD, Holcomb WR, et al. The magnitude of tissue cooling during cryotherapy with varied types of compression. J Athl Train. 2010; 45: 230-237.

[19] Matos F, Neves EB, Norte M, et al. The use of thermal imaging to monitoring skin temperature during cryotherapy: a systematic review. Infra Physics Tech. 2015; 73: 194-203.

[20] Alexander J, Rhodes D. Thermography for defining efficiency of cryotherapy modalities in sport. Temperature. 2020; 15 : 210-220.
[21] Palmieri-Smith RM, Leonard JL, Garrison JC, et al. Periphera joint cooling increases spinal reflex excitability and serum norepinephrine. Int J Neurosci. 2007; 117: 229-242.

[22] Rhodes D, Alexander J. The effect of knee joint cooling on isokinetic torque production of the knee extensors: consider ations for application. Int J Sports Phys Ther. 2018; 13: 985 992.

[23] Point M, Guilhem G, Hug F, et al. Cryotherapy induces an increase in muscle stiffness. Scand J Med Sci Sports. 2018, 28: 260-266.

[24] Cankar K, Finderle Z. Gender differences in cutaneous vascular and autonomic nervous response to local cooling. Clin Autonom Res. 2003; 13: 214-220.

[25] Kennet J, Hardaker N, Hobbs S, et al. Cooling efficiency of 4 common cryotherapeutic agents. J Athl Train. 2007; 42 343-348.

[26] Bleakley CM, Glasgow PD, Philips P, et al. For the Asso ciation of Chartered Physiotherapists in Sports and Exercise Medicine (ACPSM). Guidelines on the Management of Acute Soft Tissue Injury Using Protection Rest Ice Compression and Elevation. London: ACPSM 2011; 15-21.

[27] Duthie GM, Pyne DB, Hopkins WG, Livingstone S, Hooper SL. Anthropometry profiles of elite rugby players: quantifying changes in lean mass. Br J Sports Med. 2006; 40: 202--207.

[28] Brophy R, Silvers HJ, Gonzales T. Gender influences: the role of leg dominance in ACL injury among soccer players. Br J Sports Med. 2010; 18: 31-37.

[29] Moreira DG, Costello JT, Brito CJ., et al. Thermographic imaging in sports and exercise medicine: a Delphi study and consensus statement on the measurement of human skin temperature. J Therm Bio. 2017; 69: 155-162.

[30] Hadzic V, Tine S, Markovic G, et al. The isokinetic strength profile of quadriceps and hamstrings in elite volleyball players. Isokin Ex Sci. 2010; 18: 31-37.

[31] Cohen J. Statistical Power Analysis for the Behavioural Sci ences. $2^{\text {nd }}$ ed. Hillsdale NJ: Lawrence Erlbaum Associates 1998.

[32] Algafly AA, George KP. The effect of cryotherapy on nerve conduction velocity, pain threshold and pain tolerance. Br J Sports Med. 2007; 41: 365-369.

[33] Hawkins J, Miller KC. The importance of target tissue depth in cryotherapy application. J Athl Enhancement. 2012; 1: 1-4.

[34] Dvir Z. Clinical applicability of Isokinetics. A review. Clin Biomech. 1991; 6: 133-144.

[35] Ayala F, De Ste Croix M, Sainz de Baranda P, et al. Absolute reliability of isokinetic knee flexion and extension measurements adopting a prone position. Clin Phys and Func Imag 2012; 33: 45-54

[36] Warren T, McCarty E, Richardson A, et al. Intra-articular knee temperature changes. Am J Sports Med. 2004; 32: 441-445. 